\title{
La evolución del logotipo en el programa de mano Metro-Goldwyn-Mayer
}

\author{
Dr. James Willis García-Talavera
}

Universidad de La Laguna

\begin{abstract}
RESUMEN
El presente artículo analiza, desde una perspectiva multidisciplinar, la evolución del logotipo de una de las principales productoras y distribuidoras de Hollywood, Metro-GoldwynMayer, durante sus llamados "años dorados" (1931-1941), utilizando el programa de mano como fuente primaria de la investigación. Tras un minucioso estudio, el programa de mano se revela como un peculiar método de publicidad cinematográfica que, rebasando los límites de su función originaria, se puede convertir en modelo de expresión artística, coyuntural elemento propagandístico, archivo visual de imágenes perdidas o auténtico objeto de culto para coleccionistas.
\end{abstract}

\section{ABSTRACT}

This article analyze, from an interdisciplinary perspective, the course of the advertising emblem of one of Hollywood's main producers and distributors, MGM, during the socalled Golden Years (1931/1941) using the program as a primary source of investigation. After a detailed study, the program reveals itself as a peculiar method of cinema advertising which, exceeding the limits of its original function, can become a model of art expression, structural advertising element, a visual file of lost images or real cult object for collectors.

Palabras claves: Metro-Goldwyn-Mayer/Programa de mano/Logotipo/Publicidad cinematográfica.

Key words: Metro Goldwyn-Mayer/Program/Emblem/Film advertising.

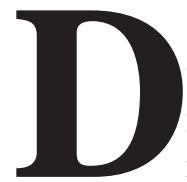

entro de la actual sociedad de la información, es unánimemente admitido, y jamás ha planteado dudas, el hecho de que la publicidad sea la industria más representativa de nuestro tiempo, que constituya una realidad global en auge, y que influya - cada vez más- en todos los aspectos de nuestra vida. Y no es menos cierto que la cinematografía, nacida casi al mismo tiempo que la publicidad, a finales del siglo XIX, responde igualmente a esas afirmaciones. 
Pero, aun teniendo en cuenta sus diferentes fundamentos, actividades y objetivos, ambas disciplinas están íntimamente vinculadas por un nexo de reciprocidad. La industria cinematografía, del mismo modo que dirigió desde un principio su tradicional maquinaria a producir bobinas y bobinas de celuloide, siempre se ha servido de una industria auxiliar, tan importante como la primera o principal, dedicada a la creación de sugestivos reclamos: fotografías, postales, affiches, guías de lanzamiento, carteles y programas de mano.

Estos peculiares objetos publicitarios fueron diseñados dentro de un específico esquema semiológico híbrido, inestable y laxo, propiciado por una multitud de combinaciones factibles entre texto e imagen, así como entre los diferentes significantes y sus respectivos constituyentes.

Siguiendo la segmentación estructural de Adam y Bonhomme (2000), podemos comprobar que el programa de mano presenta una naturaleza semiótica mixta, que hunde sus orígenes en dos grandes tradiciones: la de la escritura compacta y calibrada, propia del libro en el más amplio sentido, y la de la ilustración artesanal y estética, en general. De este modo, este singular modelo de publicidad se fundamenta en un sistema doble de principios antagonistas, el icónico y el verbal, donde imagen y texto coexisten como elementos bien diferenciados y, en cierto modo, enfrentados u opuestos. Junto a estos dos significantes del discurso publicitario, como elemento casi indispensable de cualquier programa de mano, se sitúa el logotipo como símbolo o emblema característico de la productora.

Metro-Goldwyn-Mayer, empresa líder del sector durante varias décadas, estaba obligada a presentar un logotipo que respondiera al prestigio consolidado en las pantallas de exhibición, sirviendo como aval o sello de garantía de la alta calidad del producto promocionado: la película en cuestión. De ahí deriva la gran importancia otorgada a un simple emblema que, desde la perspectiva estrictamente publicitaria, puede ser considerado como un significante publicitario que oscila entre el lenguaje y el diseño.

Partiendo del mismo idiolecto que el eslogan, el logotipo se diferencia porque constituye lo que los teóricos han calificado como un significante compuesto o, dicho de otra forma, una imagen-palabra. Asombroso paralelismo con el propio programa de mano, puesto que, en definitiva, esta simbiosis icónicolingüística representa la esencia tanto de uno como de otro.

La naturaleza mixta del logotipo de la Metro-Goldwyn-Mayer ha sido objeto de múltiples análisis semiológicos, como el llevado a cabo por Robert Stam, Robert Burgoyne y Sandy Flitterman-Lewis (1999):

El clásico logotipo del estudio M-G-M, con el famoso león de la Metro rodeado de una tira de película sobre la que está escrito "Ars Gratia Artis", debajo de la cual vemos una máscara con guirnaldas, colocada, a su vez, sobre las palabras "Metro Goldwyn Mayer". Las diversas superficies planas del logotipo ponen en juego elementos icónicos y simbólicos: la naturaleza descollante -el 
león- y la cultura -el lenguaje-. Pero lo simbólico está profundamente entremezclado con lo icónico. La palabra "Metro", por ejemplo, está inscrita en caracteres romanos, mientras "Mayer" está inscrita en neogóticos. La polisemia de la máscara, al tiempo que desempeña un papel mediador, evoca las máscaras clásicas tanto de la tragedia como de la comedia (y por tanto, la elevada dignidad del arte clásico) y el retrato racista de las películas de safaris (evocado por los rasgos estereotipadamente africanos de las máscaras y los labios gruesos). La construcción híbrida de la máscara señala, por tanto, por un lado en dirección hacia la artisticidad clasista evocada por el "Ars Gratia Artis" y por otro, al poder primitivo del león. Reunidos, los signos icónicos y simbólicos designan las amplias orientaciones de las producciones de la M-G-M: aventuras primitivas, tragicomedias sentimentales y épicas espectaculares.

En un principio, el logotipo Metro-Goldwyn-Mayer empieza teniendo una estructura eminentemente lingüística, ordenado en torno al nombre de la marca. Se puede observar que, en los primeros carteles y programas de mano elaborados tras la creación de la compañía en 1925, este significante quedaba reducido a una simple inscripción que contenía el nombre de la empresa en letras mayúsculas.

Al examinar los modelos dobles, trípticos y desplegables del programa de mano correspondiente a la película Ben-Hur (Ben-Hur), de Fred Niblo, el gran éxito de la temporada de 1926, se distingue en el borde inferior de los mismos, el rótulo: «producción METRO-GOLDWYN-MAYER». Además de estos variados formatos, existe un modelo troquelado de exquisita factura, que representa la cuadriga del personaje central, con un ingenioso dispositivo que permite mover el cuerpo del auriga. En el extremo inferior de este programa de mano se puede leer, igualmente, la mencionada frase que alude a la producción del filme, junto a otra que, a su lado, hace referencia a la distribución.

Tal como se comprueba en los ejemplos citados, es precisamente un elemento del constituyente mínimo del significante lingüístico, esto es, la marca de la empresa productora, el que ejerce las funciones propias del logotipo. Por tanto, llegamos a la conclusión de que en una primera época no existía el significante compuesto como tal.

Posteriormente, con el paso de las temporadas -de forma paralela a la propia evolución, en su conjunto, del programa de mano-, el logotipo experimentó una progresiva transformación que lo adaptó a los continuos cambios de diseño. Así, en una primera fase se sucedieron muchos modelos diferentes hasta, tras la estandarización de formatos, convertirse en un logotipo icónico casi puro. Después de esta homogeneización, nunca se llegaría a conseguir la iconicidad total del logotipo, debido a la persistencia residual de las iniciales M-G-M, vestigio de su pomposo pasado.

A finales de los años veinte y principios de los treinta, el logotipo empieza a perder la exclusividad de su base lingüística, constituyéndose como verdadero 
significante compuesto al incorporar también un componente icónico, que no es otro que la representación de su mascota, el león.

Esta base icónica también experimenta una serie de cambios, que la hacen evolucionar desde la ostentación y la exuberancia, hasta la más completa sencillez y esquematización. Esta transformación conlleva que el logotipo pase de ser esencialmente figurativo, representando unos elementos naturales como los rasgos felinos de su mascota, hasta llegar a adoptar, tras la estandarización de formatos, una apariencia no figurativa o geométrica pura. La metamorfosis se consuma en los años cincuenta, cuando, aproximándose a la abstracción, se pone en juego la más simplista de las composiciones geométricas: un óvalo que encierra las iniciales M-G-M.

Circunscribiéndonos al período analizado en nuestra investigación -los comienzos del cine sonoro- nos percatamos de que, excluyendo el último de los modelos descritos, prácticamente todas las formas evolutivas del logotipo se pueden apreciar en los programas de mano correspondientes a tan sólo diez años de la historia de la productora.

Esquemáticamente, entre 1931 y 1941, distinguimos seis grandes estadios evolutivos del logotipo Metro-Goldwyn-Mayer bien diferenciados, desde los puntos de vista gráfico y publicitario -no tan bien, desde una perspectiva cronológica, al existir una concurrencia puntual de varios modelos-, que tienen una base fundamentalmente icónica, en mayor o menor medida, pero siempre preponderante respecto al soporte lingüístico.

Es muy importante puntualizar, y reiterar, que, dentro de esta sucesión de fases experimentadas en la transformación del logotipo, no existe una completa separación e individuación de modelos; por tanto, la aparición de un nuevo prototipo no implica necesariamente que el anterior, o anteriores, quedase fuera de circulación. Así, por ejemplo, en el periodo de transición que antecedió a la definitiva estandarización de formatos, coexistieron logotipos cuadrados y figurativos mixtos con geométricos que incluían círculos, circunferencias de diferentes tamaños, elipses, cintillas y algún rectángulo. No obstante, lo que queda demostrado, sin ningún tipo de duda, es la evidente e imparable progresión del logotipo, desde la iconicidad figurativa pura hasta la geometría más absoluta.

Los seis grandes modelos diferenciados, de una forma esquemática, son:

1. Figurativo puro.

2. Figurativo puro perfeccionado.

3 . Figurativo puro en color.

4. Icónico mixto con base lingüística.

5. Icónico mixto con base lingüística simplificado.

6. Geométrico puro. 
1) El primero de estos modelos, que comienza a implantarse en la temporada de 1931-1932 -en la práctica, limitado a los programas de mano de esa campaña-, es un logotipo eminentemente icónico, acompañado de una escasa base lingüística, representada por la divisa de la productora: ars gratia artis. Dentro de esa predominante iconicidad, es figurativo puro porque se fundamenta en la presentación de la silueta de la mascota de la Metro, el león, sin incorporar elemento geométrico alguno, como ocurriría en modelos posteriores.

Durante esa temporada se impuso un modelo determinado de programa de mano doble, en formato apaisado de 96 × $275 \mathrm{~mm}$, que solía contener uno o más de estos logotipos. Estos se encontraban ubicados tanto en el interior como en el reverso o contraportada, y casi siempre en escrupulosa disposición simétrica.

Tal es el caso de los programas de mano de las películas: Inspiración (Inspiration), de Clarence Brown; La pecadora (The Easiest Way), de Jack Conway; El hijo pródigo (The Prodigal), de Harry Pollard; Danzad, locos, danzad (Dance, Fools, Dance), de Harry Beaumont; El diablillo de la casa (It's a Wise Child) y Papá solterón (The Bachelor Father), de Robert Z. Leonard; Gordas y flacas (Reducing), de Charles F. Reisner, y Pagada (Paid), de Sam Wood, todas producidas en 1931.

También de los correspondientes a producciones del año anterior, pero estrenadas durante esa temporada, como: Flor de pasión (Passion Flower), de William C. de Mille; La divorciada (The Divorcee), Estudiantina (In Gay Madrid), y Seamos alegres (Let Us Be Gay), de Robert Z. Leonard; Enfermeras de guerra (War Nurse), de Edgar Selwyn; Luz de Montana (Montana Moon), de Mal St. Clair; Música y besos (A Lady's Morals), de Sidney Franklin; Billy The Kid (Billy The Kid), de King Vidor; Trader Horn (Trader Horn), de W. S. van Dyke; Madame Satan (Madam Satan), de Cecil B. de Mille; Amor rabioso (Love in the Rough), de Charles Reisner, y Sevilla de mis amores (The Singer of Seville), dirigida por el actor Ramón Novarro.

También se utilizó este formato en los programas de mano de las versiones en habla castellana de producciones M-G-M, como: De bote en bote y Monerías, de James Parrott; Cheri-Bibi -en singular formato desplegable- y La mujer X, de Carlos Borcosque; En cada puerto un amor, de Marcel Silver; Su última noche, de Chester Franklin; Politiquerías y Los calaveras, de James W. Horne; El proceso de Mary Dugan, de Marcel De Sano, y El presidio, de Nick Grinde, que también se estrenó durante esa temporada.

Asimismo, es importante destacar que para la reposición de Ben-Hur (Ben$H u r)$, de Fred Niblo, que, con sonido sobreañadido, se llevó a cabo en ese año, se diseñó un tipo especial de programa de mano doble en el que, igualmente, se insertó este logotipo figurativo puro. 
2) Coincidiendo con la grandilocuente campaña organizada para la celebración del denominado año Metro, en la temporada 1932-1933 se diseñó un nuevo logotipo que, con el paso de los años, se distinguiría por su extraordinaria belleza plástica -reflejo del esplendor del mejor momento de la productora- e indudable atractivo visual.

Atendiendo a los constituyentes del discurso publicitario de este nuevo logotipo, se advierte que, del mismo modo que el anterior, es enteramente figurativo, nada geométrico y con un componente lingüístico que es casi imperceptible. Este nuevo modelo hace gala de un perfeccionista diseño, elaborado con plumilla, explota eficazmente la silueta del león, en disposición frontal, y la envuelve con un fragmento de celuloide arrollado que ejerce las funciones de enmarque y realce de la efigie de la famosa mascota. En la parte superior del mismo se puede leer la divisa ars-gratia-artis, pero -rompiendo la tradición- no refleja el nombre de Metro-Goldwyn-Mayer.

Podemos afirmar, sin temor a equivocarnos, que, desde la perspectiva artística, es el modelo más exquisito de todos los creados en los años dorados de la productora del león rugiente.

Este logotipo se encuentra en los programas de mano dobles de las películas: Amor en venta (Possessed), Alma libre (A Free Soul), Canción de oriente (The Son-Daughter), Letty Lynton (Letty Lynton), Emma (Emma) y Vuelo nocturno (Night Flight), de Clarence Brown; Gran Hotel (Grand Hotel), de Edmund Goulding; Tú eres mío (Hold Your Man) y Juventud triunfante (Huddle), de Sam Wood; Amores en Hollywood (Going Hollywood), de Raoul Walsh; Los seis misteriosos (The Secret Six), de George Hill; Susan Lenox (Susan Lenox, her Fall and Rise) y Peggy de mi corazón (Peg O'My Heart), de Robert Z. Leonard; Tierra de pasión (Red Dust) y Polvorilla (Bombshell), de Victor Fleming; Rosa de medianoche (Midnight Mary), de William Wellman; El campeón (The Champ), de King Vidor; El abuelo de la criatura (Pack Up Your Troubles), de George Marshall; Broadway y Hollywood (Broadway to Hollywood), de Willard Mack; Carne (Flesh), de John Ford; Ana la del remolcador (Tugboat Annie), de Mervyn LeRoy; Bajo el cielo de Cuba (Cuban Love Song), de W. S. van Dyke; Bellezas a la venta (Beauty for Sale), de Richard Boleslawski; Cuando hace falta un amigo (When a Feller Needs a Friend), de Harry Pollard; De pura sangre (Sporting Blood), de Charles Brabin; El prófugo (The Squaw Man), de Cecil B. de Mille; Justicia divina (Payment Deferred), de Lothar Mendes; La pelirroja (Red-Headed Woman), de Jack Conway; Mata Hari (Mata Hari), de George Fitzmaurice; Vidas intimas (Private Lives), de Sidney Franklin, y Polly la chica del circo (Polly of the Circus), de Alfred Santell.

Como se puede comprobar, la práctica totalidad de estos filmes son producciones de la temporada 1932-1933, o bien de la anterior, pero estrenados en España durante el año Metro. 
Encontramos este singular logotipo en el programa troquelado de El pecado de Madelon Claudet (The Sin of Madelon Claudet), de Edgar Selwyn, y en el correspondiente a la reposición de El gran desfile (The Big Parade), dirigida por King Vidor en 1925.

También en tarjetas monocromas, formato 138 x $89 \mathrm{~mm}$, como las correspondientes a las películas Una noche en el Cairo (The Barbarian) y Tú eres mío (Hold Your Man), de Sam Wood; Tierra de pasión (Red Dust), de Victor Fleming; La máscara de Fu Manchú (The Mask of Fu Manchu), de Charles Brabin; Piernas de perfil (Speak Easily), de Edward Sedgwick; Tempestad al amanecer (Storm at Daybreak), de Richard Boleslawski, y La llama eterna (Smilin' Through), de Sidney Franklin.

3) El tercer modelo, diseñado para la temporada siguiente, es tan icónico y figurativo como el anterior y, de hecho, ambos son muy similares, si bien éste alcanza un menor nivel estético. La diferencia entre ellos estriba en que éste último exhibe la imagen coloreada del león en posición lateral -pierde fuerza de la mirada del animal- y en que su diseño no está tan bien elaborado como el primero, quizá por no haber sido delineado a plumilla.

Paradójicamente, pese a la voluntad de perfeccionamiento y a estar más alejado en el tiempo, se asemeja más al modelo embrionario de 1931 que al incomparable prototipo de la temporada precedente.

Estas circunstancias marcaron la escasa continuidad y subsistencia que tuvo este modelo. No obstante, se puede encontrar en los programas de mano dobles de películas tan importantes como La hermana blanca (The White Sister), de Victor Fleming; Como tú me deseas (As You Desire Me), de George Fitzmaurice; Honduras de infierno (Hell Bellow), de Jack Conway, o Vivamos hoy (Today We Live), de Howard Hawks.

4) El siguiente modelo que fue puesto en circulación, coexistiendo por breve espacio de tiempo con algunos de los anteriores, incorpora una base lingüística en forma de leyenda bajo la figura del león, que hace referencia al nombre de MetroGoldwyn-Mayer; y, además, un elemento geométrico -ejerce funciones de aislante y, al mismo tiempo, de enaltecedor-, representado por un marco cuadrado, de ahí su denominación como icónico mixto.

Lo encontramos en los programas dobles correspondientes a las películas Margarita Gautier (Camille) y David Copperfield (David Copperfield), de George Cukor; Ana Karenina (Anna Karenina) y Encadenada (Chained), de Clarence Brown; Había una vez dos héroes (Babes in Toyland), de Charles Rogers; Piloto de pruebas (Test Pilot) y La indómita (Reckless), de Victor Fleming; Busco un millonario (The Girl from Missouri), El cuarto $n^{\circ} 309$ (One New York Night) y La novia alegre (The Gay Bride), de Jack Conway; El acorazado misterioso (Murder 
in the Fleet), de Edward Sedgwick; Entre el amor y la muerte (Only Eight Hours) y La fugitiva (Woman Wanted), de George B. Seitz; Jugando a la misma carta (Personal Property) -logotipo extrañamente coloreado-, ;Yo vivo mi vida! (I Live My Life) y El enemigo público $n^{o} 1$ (Manhattan Melodrama), de W. S. van Dyke; El gong de la victoria (The Crowd Roars) y El último pagano (Last of the Pagans), de Richard Thorpe; Sequoia (Sequoia), de Chester Franklin; La espía de Castilla (The Firefly), El gran Ziegfeld (The Great Ziegfeld), Sola contra el mundo (Outcast Lady) y El escándalo del día (After Office Hours), de Robert Z. Leonard; El héroe público $n^{o} 1$ (Public Hero Number One), de J. Walter Ruben; El vagón de la muerte (Murder in the Private Car), de Harry Beaumont; El ángel negro (The Shopworn Angel), de H. C. Potter; La viuda alegre (The Merry Widow), de Ernst Lubitsch; El billete premiado (The Winning Ticket), de Charles Reisner; Vanessa (Vanessa, Her Love Story), de William K. Howard; La voz que acusa (The Murder Man), de Tim Whelan; Muñecos infernales (The Devil Doll), de Tod Browning; Esposados y desposados (Pursuit) y El crimen del casino (The Casino Murder Case), de Edwin L. Marin; Sangre de circo (O'Shaughnessy's Boy) y El velo pintado (The Painted Veil), de Richard Boleslawski; Dos fusileros sin bala (Bonnie Scotland), de James W. Horne; La estropeada vida de Oliverio VIII (Oliver the Eight), de L. French y Charles Rogers; En los tiempos del vals (The Night is Young), de Dudley Murphy, y Jaque al rey (Whipsaw), de Sam Wood.

En las tarjetas coloreadas de El enemigo público $n^{\circ} 1$ (Manhattan Melodrama), El refugio (Hide-Out) y La cena de los acusados (The Thin Man), de W. S. van Dyke; Honduras de infierno (Hell Bellow), Busco un millonario (The Girl From Missouri) y Viva Villa (Viva Villa!), de Jack Conway; Hombres en blanco (Men in White), de Richard Boleslawski; Así ama la mujer (Sadie McKee), de Clarence Brown; Las vírgenes de Wimpole Street (The Barrets of Wimpole Street), de Sidney Franklin; Fra Diavolo (The Devil's Brother), de Hal Roach y Charles Rogers; Tarzán y su compañera (Tarzan and his Mate), de Cedric Gibbons y Jack Conway; El misterioso Sr. X (The Mystery of Mr. X), de Edgar Selwyn, y Alma de bailarina (Dancing Lady), de Robert Z. Leonard.

Es digna de mención la aparición de una tarjeta coloreada, una de las correspondientes al filme La reina Cristina de Suecia (Queen Christina), de Rouben Mamoulian, en la que, de forma desacostumbrada, se inserta este logotipo sin el usual elemento geométrico acompañante -el marco cuadrado-, una excepción que provoca la transformación del significante compuesto, que deja de ser icónico mixto y se convierte en figurativo puro.

Hacemos constar la presencia de este modelo de logotipo en una serie de programas de mano en color, escasa o variablemente definidos -por lo general, elaborados usando sólo tres tonalidades cromáticas-, pertenecientes a la época de transición previa a la estandarización, como son los de las películas Las vacaciones del juez Harvey (You're Only Young Once), de George B. Seitz; Un día en las 
carreras (A Day at the Races), de Sam Wood; Un corazón y una copa (The Good Old Soak), de J. Walter Ruben; Dos pares de mellizos (Our Relations), de Harry Lachman, y las dos variantes de La melodía de Broadway (Broadway Melody of 1936), de Roy del Ruth, una producción de 1935 pero que en nuestro país se estrenó con gran retraso debido a innumerables problemas de censura.

5) La quinta modalidad de logotipo es una variación del anterior -quizá, poco afortunada, ya que pierde encanto visual y no aporta novedades interesantes-, que mantiene intacta su condición de icónico mixto. Conserva el elemento geométrico ideado para ejercer de separador del entorno y para realzar el prestigio empresarial, y modifica ligeramente la base linguiística, aumentando el cuerpo de los tipos de Metro-Goldwyn-Mayer.

Está menos cuidado estéticamente, aunque, dentro de la grosería de trazos, sea algo más llamativo. Lo verdaderamente importante es la constatación de la clara e imparable tendencia a la simplificación que, como ya ha sido comentado, experimentarían, paralelamente, tanto el logotipo como el programa de mano en su conjunto, para desembocar en la más absoluta sencillez y economía de recursos.

En la época de transición que precedió a la definitiva homogeneización de formatos, este logotipo fue bastante utilizado y lo podemos encontrar en numerosos programas de mano, así como en diferentes modelos: dobles y trípticos formato estándar, dobles de dimensiones reducidas, troquelados, desplegables, etc.

En dobles y trípticos, como los de las películas Rebelión a bordo (Mutiny on the Bounty), de Frank Lloyd; Entre esposa y secretaria (Wife versus Secretary), de Clarence Brown; Mares de China (China Seas), de Tay Garnett; Suzy (Suzy), de George Fitzmaurice; La esposa de su hermano (His Brother's Wife) y Rose Marie (Rose Marie), de W. S. van Dyke; La melodía de Broadway (Broadway Melody of 1936), Nacida para la danza (Born to Dance) y La melodía de Broadway de 1938 (Broadway Melody of 1938), de Roy del Ruth; Joaquín Murrieta (Robin Hood of El Dorado) y Una chica de provincias (Small Town Girl), de William Wellman; Una pareja invisible (Topper), de Norman Z. McLeod; Furia (Fury), de Fritz Lang; El último gángster (The Last Gangster), de Edward Ludwig; Primavera (Maytime) y Ciudad del oro (The Girl of the Golden West), de Robert Z. Leonard; Bajo el manto de la noche (Undercover of Night) y La sombra del hampa (Exclusive Story), de George B. Seitz; Flor de arrabal (Riffraff), de J. Walter Ruben; Adán sin Eva (Petticoat Fever), de George Fitzmaurice; Su primera escapada (Tough Guy), de Chester M. Franklin; Historia de dos ciudades (A Tale of Two Cities), de Jack Conway; La fuga de Tarzán (Tarzan escapes), de Richard Thorpe; Dos pares de mellizos (Our Relations), de Harry Lachman; Un par de gitanos (A Bohemian Girl), de J. W. Horne y Charles Rogers; Quesos y besos (Swiss Miss), de John G. Blystone; Cadetes del mar (Navy Blue and Gold), Una noche en la ópera (A Night at the Opera) y Un día en las carreras 
(A Day at the Races), de Sam Wood, y Las manos de Orlac (Mad Love), de Karl Freund.

En dobles de pequeño formato, como los de los filmes Romeo y Julieta (Romeo and Juliet), de George Cukor; El juez Harvey y sus hijos (Judge Hardy's Children), de George B. Seitz; El demonio es un pobre diablo (The Devil is a Sissy), de Woody Strong van Dyke; Un yanqui en Oxford (A Yank at Oxford), de Jack Conway; Un corazón y una copa (The Good Old Soak) y El tigre de Arizona (The Bad Man of Brimstone), de J. Walter Ruben, y Horizontes de gloria (Lord Jeff), de Sam Wood.

$\mathrm{Y}$ en otros modelos más selectos y singulares como el apaisado monocromo de Una mujer difamada (Libeled Lady), de Jack Conway; el grande semitroquelado de Rosalie (Rosalie), de W. S. van Dyke, o el desplegable de Ana Karenina (Anna Karenina), de Clarence Brown.

En lo que respecta a los programas de mano sencillos, lo más destacable es el hecho de que el elemento geométrico, el marco cuadrado, aparte de aislar el logotipo de su entorno, solía estar relleno de un color predeterminado y concreto -preferentemente rojo o azul, y raramente el negro-, que lo hacía destacar a simple vista.

Encontramos logotipos cuadrados de color rojo en los programas de mano sencillos de muchas películas estrenadas en la época de transición, como por ejemplo: Piloto de pruebas (Test Pilot), de Victor Fleming; Horizontes de gloria (Lord Jeff), de Sam Wood; Rosalie (Rosalie), Jugando a la misma carta (Personal Property) y El demonio es un pobre diablo (The Devil is a Sissy), de W. S. van Dyke; Ha desaparecido un hombre (Sinner Take All), de Errol Taggert; El tigre de Arizona (The Bad Man of Brimstone), de J. Walter Ruben; El ángel negro (The Shopworn Angel), de H. C. Potter; La fuga de Tarzán (Tarzan Escapes), de Richard Thorpe, y Un yanqui en Oxford (A Yank at Oxford), de Jack Conway.

Azules, en los programas de mano sencillos correspondientes a los filmes: Honor de familia (A Family Affair), de George B. Seitz; Forja de hombres (Boys Town), de Norman Taurog; Una pareja invisible (Topper), de Norman Z. McLeod -una de sus dos variantes-; Cadetes del mar (Navy Blue and Gold), de Sam Wood; Una mujer difamada (Libeled Lady), de Jack Conway; Quesos y besos (Swiss Miss), de John G. Blystone, y El gong de la victoria (The Crowd Roars), de Richard Thorpe.

Y las excepciones en negro, en los de los de las películas: La vuelta de Arsenio Lupin (Arsene Lupin Returns), de George Fitzmaurice, y Nacida para la danza (Born to Dance), de Roy del Ruth.

La utilización de este logotipo en programas sencillos no queda circunscrita, únicamente, a películas de la época de transición previa a la estandarización, porque también lo encontramos en tarjetas coloreadas de producciones anteriores como: Tierra de pasión (Red Dust) y La hermana blanca (The White Sister), de 
Victor Fleming; Había una vez dos héroes (Babes in Toyland), de Charles Rogers; La llama eterna (Smilin' Through), de Sidney Franklin; La estropeada vida de Oliverio VIII (Oliver the Eighth), de L. French y Charles Rogers -excepción figurativa pura, al prescindir del elemento geométrico-; Mademoiselle Doctor (Stamboul Quest), de Sam Wood, y Una fiesta en Hollywood (Hollywood Party), de Richard Boleslawski.

También en aquellos programas de mano de la temporada de 1933 que se adelantaron a su tiempo y mostraron el diseño que iba a predominar en todos los programas sencillos de las décadas siguientes. Hablamos de las tarjetas correspondientes a cinco filmes de bajo presupuesto: De mujer a mujer (When Ladies Meet), de Harry Beaumont; Marido y Cía (Another Language), de Edward H. Griffith; El futuro es nuestro (Looking Forward), de Clarence Brown; Vuelta atrás al reloj (Turn Back the Clock), de Edgar Selwyn, y En la pendiente (This Side of Heaven), de William K. Howard.

6) El sexto y último modelo es el que perduraría durante un mayor espacio de tiempo tras la determinante y definitiva estandarización del programa de mano. Es un logotipo que, icónicamente, puede ser definido como geométrico puro, y que, a su vez, posee la más elemental y rudimentaria de las bases lingüísticas, representada, únicamente, por las letras del nombre de la productora: M-G-M. Se trata de un esquemático significante compuesto de naturaleza redonda, coloreado o transparente, y bordeado por una delgada circunferencia de la misma tonalidad cromática que las iniciales que engloba.

Limitándonos a las películas comprendidas dentro del acotamiento temporal de nuestra investigación -e incluyendo las reposiciones-, podemos encontrar este logotipo en un amplísimo grupo de programas de mano sencillos: La hora radiante (The Shining Hour), de Frank Borzage; Margarita Gautier (Camille), Cena a las ocho (Dinner at Eight), Romeo y Julieta (Romeo and Juliet) -dos variantes-, Historias de Filadelfia (The Philadelphia Story), Un rostro de mujer (A Woman's Face) y La mujer de las dos caras (Two-Faced Woman), de George Cukor; San Francisco (San Francisco), Ella, él y Asta (After the Thin Man), Otra reunión de acusados (Another Thin Man), María Antonieta (Marie Antoinette), En este mundo traidor (It's a Wonderful World), Te quiero otra vez (I Love You Again), Un nuevo amor de Andrés Harvey (Andy Hardy Gets Spring Fever), Enamorados (Sweethearts), Esta mujer es mía (I Take This Woman) y Alma en la sombra (Rage in Heaven), de W. S. van Dyke; La hermana blanca (The White Sister), La indómita (Reckless), Capitanes intrépidos (Captains Courageous) -dos variantes-, El extraño caso de Dr. Jekyll (Dr. Jekyll and Mr. Hyde), El mago de Oz (The Wizard of $\mathrm{Oz}$ ) y Lo que el viento se llevó (Gone with the Wind), de Victor Fleming; Sucedió en China (Too Hot To Handle), Honduras de infierno (Hell Bellow), Historia de dos ciudades (A Tale of Two Cities), Mi marido está loco 
114 La evolución del logotipo en el programa de mano Metro-Goldwyn-Mayer

(Love Crazy) -dos variantes-, Fruto Dorado (Boom Town) y Quiero a este hombre (Honky Tonk), de Jack Conway; La tragedia de la Bounty (Mutiny on the Bounty) -dos variantes-, de Frank Lloyd; Camarada X (Comrade X), de King Vidor; El gran Ziegfeld (The Great Ziegfeld), Se llevó mi corazón (Broadway Serenade), Luna Llena (New Moon), Ciudad del oro (The Girl of the Golden West) -dos variantes-, Primavera (Maytime), La espía de Castilla (The Firefly) -dos variantes-, Más fuerte que el orgullo (Pride and Prejudice) y Cuando ellas se encuentran (When Ladies Meet), de Robert Z. Leonard; De corazón a corazón (Blossoms in the Dust) y El puente de Waterloo (Waterloo Bridge), de Mervyn LeRoy; La nueva melodía de Broadway (Broadway Melody of 1940) -dos variantes-, La ciudad de los muchachos (Men of Boys Town) y El joven Edison (Young Tom Edison), de Norman Taurog; María Walewska (Conquest), Ayer como hoy (Ah, Wilderness), Edison, el hombre (Edison the Man) y No puedo vivir sin ti (Come Live With Me), de Clarence Brown; El último gángster (The Last Gángster), de Edward Ludwig; Ninotchka (Ninotchka) -dos variantes-, La viuda alegre (The Merry Widow) y El bazar de las sorpresas (The Shop Around the Corner), de Ernst Lubitsch; Al caer la noche (Night Must Fall), Tarzán y su hijo (Tarzan Finds a Son), El tesoro de Tarzán (Tarzan Secret Treasure) -dos variantes-, Doble boda (Double Wedding) y Puño de hierro (20-Mule Team), de Richard Thorpe; Uña y carne (Stablemates) y Juventud deportiva (Huddle), de Sam Wood; Laurel y Hardy en el Oeste (Way Out West), de James W. Horne; Vivamos hoy (Today We Live), de Howard Hawks; Invitación peligrosa (Fast and Loose), de Edwin L. Marin; Mata Hari (Mata Hari), Suzy (Suzy), La vuelta de Arsenio Lupin (Arsene Lupin Returns) - una de sus dos variantes- y El secreto del candelabro (The Emperor's Candlesticks), de George Fitzmaurice; La reina Cristina de Suecia (Quenn Christina) -tres variantes-, de Rouben Mamoulian; La llama eterna (Smilin' Through), de Sidney Franklin; Tempestad al amanecer (Storm at Daybreak), de Richard Boleslawski; No más mujeres (No More Ladies) -dos variantes-, de Edward H. Griffith; El juez Harvey y sus hijos (Judge Hardy's Children), Andrés Harvey tenorio (Andy Hardy Meets Debutante) -dos variantes-, Andrés Harvey millonario (Love Finds Andy Hardy) y Andrés Harvey se enamora (The Hardys Ride High), de George B. Seitz; Armonías de juventud (Strike Up the Band) e Hijos de la farándula (Babes in Arms), de Busby Berkeley; Honolulu (Honolulu), Los hermanos Marx en el Oeste (Go West) y Una tarde en el circo (Marx Brothers at the Circus), de Edward Buzzell; Una pareja invisible (Topper) -una de sus dos variantes-, de Norman Z. McLeod, y El gran vals (The Great Vals), de Julien Duvivier.

También hallamos este logotipo en una serie de programas de mano dobles, correspondientes a muchas de las películas anteriormente citadas, como: Camarada X, Fruto dorado, Lo que el viento se llevó, María Antonieta, El mago de Oz, El gran vals, La mujer de las dos caras, Historias de Filadelfia, María Walewska, 
Ella, él y Asta, Otra reunión de acusados, Enamorados, De corazón a corazón, Un rostro de mujer, Más fuerte que el orgullo, La ciudad de los muchachos, El joven Edison, Edison el hombre y El extraño caso del Dr. Jekyll.

En la gran mayoría de estos programas de mano, se armoniza la presencia de este logotipo, normalmente en el interior a color, con otro logotipo del modelo figurativo puro, que suele insertarse en la portada.

Existe un modelo preliminar, representado por una circunferencia incompleta, abierta en su extremo inferior, que se diseñó a modo de prueba cuando todavía no se había decidido cuál sería el definitivo modelo acompañante de los nuevos ejemplares estandarizados. Lo localizamos en dos programas de mano dobles, creados para el estreno de sendos filmes de la temporada 1936-1937: Honor de familia (A Family Affair), de George B. Seitz, y San Francisco (San Francisco), de Woody Strong van Dyke.

Antes de cerrar el presente artículo, y para constatar el amplio abanico de modelos existentes al margen de la precedente clasificación, estamos obligados a citar algunos casos singulares o excepciones a la regla. Son rarezas que van desde el logotipo del programa de mano doble de Romeo y Julieta (Romeo and Juliet), de George Cukor, original modelo en forma de cintilla, acorde con la ambientación medieval de la película; pasando por el modelo Art Déco insertado en el vértice superior del ejemplar doble de El refugio (Hide-Out), de W. S. van Dyke; hasta el incluido en el reverso del programa de mano -formato único- de $L a$ fugitiva (Woman Wanted), de George B. Seitz, un inusual logotipo con exclusiva base lingüística.

Independientemente del momento evolutivo y en términos generales, el logotipo de la Metro nunca dejó de formalizar y satisfacer, en su totalidad, cada uno de sus argumentativos propósitos.

La función valorizadora de la imagen empresarial fue la indeclinable constante de todos los modelos, con especial predisposición de los icónicos puros. La apropiación inmediata de la marca estuvo garantizada, desde un principio, tanto por la instantánea identificación de los genuinos rasgos del león, como por los ventajosos efectos -publicitariamente hablando- de la evocación de su concepto global, con todas las vivencias positivas asociadas que éste, presumiblemente, despertaría en la mente de cualquier espectador.

\section{Bibliografía recomendada:}

- ADAM, Jean-Michel; Bonhomme, Marc, 2000: La argumentación publicitaria. Retórica del elogio y de la persuasión, Madrid, Cátedra.

- ALCÁCER GARMENDIA, José A., 1991: Qué es: el mundo del cartel. Madrid, Ediciones Granada. 
- APARICI, Roberto; García-Matilla, Agustín, 1987: Lectura de imágenes. Madrid, Ediciones de la Torre.

- BAENA PALMA, Francisco,1994: Los programas de mano en España. Barcelona, F.B.P.

- - 1996, El cartel de cine en España. Barcelona, F.B.P.

- BARNICOAT, Joan, 1976, Los carteles, su historia y lenguaje. Barcelona, Editorial Gustavo Gili.

- BARTHES, Roland, 1957, Mythologies. París, Seuil.

- - 1982, L'Obvie et L'Obtus. París, Seuil.

- - 1989, La cámara lúcida. Barcelona, Paidós.

- - y cols, 1970, La semiología. Buenos Aires, Ed. Tiempo Contemporáneo.

- BAZIN, André, 1966, ¿Qué es el cine? Madrid, Ediciones Rialp.

- - 1989, Estética y psicología del cine. Tomo 1, Madrid, Siglo XXI España.

- BEJARANO PÉREZ, Rafael, 1986: El prospecto, propaganda cinematográfica, Málaga, Facultad de Filosofía y Letras de Málaga.

- CLARK, Eric, 1989: La publicidad y su poder. Barcelona, Planeta.

- Eames, John Douglas, 1982: The MGM Story. Londres, Sundial Publications Ltd.

- ENEL, Francoise, 1974: El cartel: lenguaje, funciones y retórica. Valencia, Editorial Fernando Torres.

- GARCÍA GUATAS, Manuel, 1993: Publicidad artística en Zaragoza. Zaragoza, Ed.Ibercaja. Colección Boira número 20.

- GARRONI, Emilio, 1975: Proyecto de Semiótica, Mensajes artísticos no verbales. Problemas teóricos y aplicados. Barcelona, Editorial Gustavo Gili.

- GIRALT-MIRACLE, Daniel, 1981: Historia social y cultural del cartel. Madrid-Barcelona, Centro de Promoción de las Artes Plásticas e Investigación de nuevas formas expresivas, Dirección General de Patrimonio Artístico, Ministerio de Cultura.

- GUBERN, Román, 1974: Mensajes icónicos en la cultura de masas. Barcelona, Lumen.

- - 1997, Historia del cine. Barcelona, Lumen.

- GUINSBERG, Enrique, 1987: Publicidad:manipulación para la reproducción. México, Plaza y Janés.

- HAY, Peter, 1991: M-G-M When Lion Roars, Nueva York, Turner.

- JARVIE, Ian C., 1974: Sociología del Cine. Barcelona, Ediciones Guadarrama.

- Julian, Inmaculada, 1976: El cartelismo y la gráfica en la guerra civil española, vanguardia artística y realidad social, 1936-1976. Barcelona, Editorial Gustavo Gili.

- KERR, Paul, 1986: The Hollywood Film Industry. Londres, Routledge and Kegan Paul.

- MARIN, Louis, 1978: Estudios semiológicos. Madrid, Alberto Corazón. 
- MARSTON SEABURY, William, 1926: The Public and the Motion Picture Industry, Nueva York, Macmillan.

- METZ, Christian, 1974a: Language and Cinema. La Haya, Mouton.

- - 1974b, Film Language. A Semiotics of the Cinema. Nueva York, Oxford University Press.

- -1979, Psicoanálisis y cine. El significante imaginario. Barcelona, Editorial Gustavo Gili.

- MILlER, Frank, 1994: MGM Posters. Atlanta, Turner Publishing.

- MOLES, Abraham, 1958: Théorie de l'information et Perception esthétique. París, Flammarion.

- - 1976, El afiche en la sociedad urbana. Buenos Aires, Paidós.

- - 1981, L'Image, communication fonctiounelle. París, Casterman.

- MORIN, Edgar, 1957: Les Stars. París, Seuil.

- - 1975, El Cine o el hombre imaginario, Barcelona, Editorial Seix Barral.

- MORRIS, Charles, 1946: Signs, Language and Behaviour. Nueva York, Prentice Hall.

- - 1994, Fundamentos de la teoría de los signos. Barcelona, Paidós.

- Panofsky, Erwin, 1985: Estudios sobre iconología. Madrid, Alianza.

- - 1986, La perspectiva como «forma simbólica». Barcelona, Tusquets.

- PARISH, James Robert, 1981: The Best of M-G-M: The Golden Years (192859), Westport, Arlington House Publishers.

- PENINOU, G., 1976: Semiótica de la publicidad. Barcelona, Gustavo Gili.

- PERICOT, Jordi, 1987: Servirse de la imagen. Un análisis pragmático de la imagen, Barcelona, Ariel.

- POWDERMAKER, Hortense, 1950: Hollywood, The Dream Factory. Boston, Little, Brown and Co.

- REBELlO, Stephen; Allen, Richard, 1988: Reel Art: Great Movie Posters from The Golden Age of Silver Screen. Nueva York, Abbeville Press.

- SATUE, Enric, 1988: El diseño gráfico. Desde los orígenes hasta nuestros días, Madrid, Alianza.

- SCHAEFFER, Jean-Marie, 1990: La imagen precaria. Madrid, Cátedra.

- SCHUDSON, Michael, 1984: Advertising: The Uneasy Persuasion. Nueva York, Basic Books.

- STAM, Robert; Burgoyne, Robert; Flitterman-Lewis, Sandy, 1999: Nuevos conceptos de la teoría del cine. Estructuralismo, semiótica, narratología, psicoanálisis, intertextualidad. Barcelona, Paidós. 